PROCEEDINGS OF THE

AMERICAN MATHEMATICAL SOCIETY

Volume 125, Number 10, October 1997, Pages 2907-2919

S 0002-9939(97)03941-5

\title{
WEAK SOLVABILITY AND WELL-POSEDNESS OF A COUPLED SCHRÖDINGER-KORTEWEG DE VRIES EQUATION FOR CAPILLARY-GRAVITY WAVE INTERACTIONS
}

\author{
DANIELLA BEKIRANOV, TAKAYOSHI OGAWA, AND GUSTAVO PONCE
}

(Communicated by J. Marshall Ash)

\begin{abstract}
An interaction equation of the capillary-gravity wave is considered. We show that the Cauchy problem of the coupled Schrödinger-KdV equation,

$$
\left\{\begin{array}{l}
i \partial_{t} u+\partial_{x}^{2} u=\alpha v u+\gamma|u|^{2} u, \quad t, x \in \mathbb{R}, \\
\partial_{t} v+\partial_{x}^{3} v+\partial_{x} v^{2}=\beta \partial_{x}\left(|u|^{2}\right), \\
u(x, 0)=u_{0}(x), v(x, 0)=v_{0}(x),
\end{array}\right.
$$

is locally well-posed for weak initial data $u_{0} \times v_{0} \in L^{2}(\mathbb{R}) \times H^{-1 / 2}(\mathbb{R})$. We apply the analogous method for estimating the nonlinear coupling terms developed by Bourgain and refined by Kenig, Ponce, and Vega.
\end{abstract}

\section{INTRODUCTION}

An interaction phenomenon between long waves and short waves has been studied in various physical situations. This phenomenon is of interest in several fields of physics and fluid dynamics: an electron-plasma, ion-field interaction [20], a diatomic lattice system [27], and a water wave theory [8]. The short wave is usually described by the Schrödinger type equation and the long wave is described by some sort of wave equation accompanied with a dispersive term. In the theory of capillary-gravity waves, Kawahara et al. [13] studied the coupled system

$$
\left\{\begin{array}{l}
i \partial_{t} S+i c_{s} \partial_{x} S+\partial_{x}^{2} S=\alpha S L, \quad t, x \in \mathbb{R}, \\
\partial_{t} L+c_{l} \partial_{x} L+\partial_{x}^{3} L+\partial_{x} L^{2}=\beta \partial_{x}|S|^{2},
\end{array}\right.
$$

where $S$ and $L$ describe short and long water waves and $\alpha, \beta, c_{s}, c_{l}$ are real constants. When the resonance condition $c_{s}=c_{l}$ holds, this equation is known as the coupled Schrödinger-KdV equation. This paper is concerned with the Cauchy problem for the following general version of the coupled Schrödinger-KdV equation, with $u_{0} \in L^{2}(\mathbb{R})$ and $v_{0} \in H^{-1 / 2}(\mathbb{R})$ :

$$
\left\{\begin{array}{l}
i \partial_{t} u+\partial_{x}^{2} u=\alpha v u+\gamma|u|^{2} u, \quad t, x \in \mathbb{R}, \\
\partial_{t} v+\partial_{x}^{3} v+\partial_{x} v^{2}=\beta \partial_{x}\left(|u|^{2}\right), \\
u(x, 0)=u_{0}(x), v(x, 0)=v_{0}(x) .
\end{array}\right.
$$

Received by the editors April 24, 1996.

1991 Mathematics Subject Classification. Primary 35Q53, 35Q55, 76B15.

Key words and phrases. Capillary-gravity wave, nonlinear Schrödinger, KdV, well-posedness. The third author was partially supported by an NSF grant. 
One of the closely related interactions is described by the following system:

$$
\left\{\begin{array}{l}
i \partial_{t} u+\partial_{x}^{2} u=u v, \quad t, x \in \mathbb{R}, \\
\partial_{t} v=\partial_{x}\left(|u|^{2}\right), \\
u(x, 0)=u_{0}(x), v(x, 0)=v_{0}(x),
\end{array}\right.
$$

which was introduced by Benney [3] (see also Yajima-Oikawa [26] and FunakoshiOikawa [8]) and both the inverse scattering method ([26], [19]) and the theory of evolution equations ([24], [18], [1]) have been applied.

Unlike the results for Benney's equation (1.3), the coupled Schrödinger-KdV equation (1.2) has been shown not to be a completely integrable system (BenirovBurtsev [2]). Therefore the solvability of (1.2) is dependent upon the method of evolution equations. If we assume the initial data $\left(u_{0}, v_{0}\right)$ is sufficiently smooth, we have time global well-posedness for (1.2). More specifically M. Tsutsumi [23] showed that for $\left(u_{0}, v_{0}\right) \in H^{m+\frac{1}{2}}(\mathbb{R}) \times H^{m}(\mathbb{R})$ for $m=1,2,3, \ldots$ the coupled system (1.2) is globally well-posed in $H^{m+\frac{1}{2}}(\mathbb{R}) \times H^{m}(\mathbb{R})$.

On the other hand, it has been recently realized that related single dispersive equations are well-posed even with weaker initial data. For nonlinear Schrödinger equations, $L^{2}$ - well-posedness has been shown by Y. Tsutsumi [25], Kato [12], Cazenave-Weissler [7] and for negative Sobolev spaces by Kenig-Ponce-Vega [16]; for the KdV equation, $L^{2}$ well-posedness was shown by Bourgain [4], [5], [6] and for negative Sobolev spaces by Kenig-Ponce-Vega [14] (for an expository summary, see [9]). If we establish the well-posedness in a weaker space, then the interaction model is valid for more singular waves than continuous solutions. Moreover, for the case of Benney's equation (1.3), it has been shown that for weaker initial data, $\left(u_{0}, v_{0}\right) \in H^{\epsilon}(\mathbb{R}) \times L^{1 / \epsilon}(\mathbb{R})$ for $\epsilon>0$ is well-posed (Bekiranov-Ogawa-Ponce [1]). Therefore, it is natural to ask if the coupled system (1.2) is also well-posed for weaker initial data $\left(u_{0}, v_{0}\right)$. To consider weak solutions, we reduce the system (1.2) to the following integral equation:

$$
\begin{aligned}
& u(t)=U(t) u_{0}-i \int_{0}^{t} U\left(t-t^{\prime}\right)\left\{\alpha v\left(t^{\prime}\right) u\left(t^{\prime}\right)+\gamma\left|u\left(t^{\prime}\right)\right|^{2} u\left(t^{\prime}\right)\right\} d t^{\prime}, \\
& v(t)=V(t) v_{0}+\int_{0}^{t} V\left(t-t^{\prime}\right)\left\{\beta \partial_{x}\left|u\left(t^{\prime}\right)\right|^{2}-\partial_{x} v\left(t^{\prime}\right)^{2}\right\} d t^{\prime},
\end{aligned}
$$

where $U(t)=e^{i \partial_{x}^{2} t}$ and $V(t)=e^{-\partial_{x}^{3} t}$ are the linear Schrödinger and Airy unitary groups, respectively.

The well-posedness for the single nonlinear Schrödinger equation in the Sobolev space with negative exponents has been obtained up to $H^{-\frac{1}{2}+\varepsilon}(\mathbb{R})$, and for the $\mathrm{KdV}$ equation, $H^{-\frac{3}{4}+\varepsilon}(\mathbb{R})([6]$, [14], [15]). In general, a coupled system like equation (1.2) is more difficult to handle in the same spaces as in the space the single equation is solved. The difficulty stems from antisymmetric characters of the characteristics of each linear part. Due to the dispersive term in the KdV part, however we still have room to handle well-posedness in $L^{2}(\mathbb{R}) \times H^{-1 / 2}(\mathbb{R})$.

The main method is based on the analogous argument as put forth by Bourgain ([4], [5], [6]) in the spatially periodic case and the Cauchy problem by Kenig-PonceVega ([14], [15], [16] and see also [17]). The key fact is that we use both space-time weighted norms in the phase space to see the smoothing effect of two dispersive linear equations and smoothing effects of the quadratic nonlinearities which is seen as terms of a convolution of weight potential. The heart of this technique was 
extensively developed by Kenig-Ponce-Vega in order to establish the well-posedness for the single KdV equation and the nonlinear Schrödinger equations.

Since the quadratic nonlinearities can be written as a form of convolution and the different nature of each characteristic of the linear part of the Schrödinger and $\mathrm{KdV}$ equations, we are able to avoid the difficulty of derivative loss which commonly appears to construct a weak solution.

Before stating the theorem we give the following notations. Let $\|\cdot\|_{2}$ be the $L^{2}(\mathbb{R})$ norm for space variable. Let $H^{s}(\mathbb{R})$ denote the Sobolev space with norm $\|v\|_{H^{s}}=\left\|\left(1+D_{x}\right)^{s} v\right\|_{2}$, where $D_{x}^{\alpha}$ stands for the fractional derivative by the Riesz potential in the space variable. We denote by $L_{t}^{p}\left(L_{x}^{q}\right)(1<p, q \leq \infty)$ the Banach spaces $L^{p}\left(\mathbb{R}: L^{q}(\mathbb{R})\right)$ for variables $t$ and $x$ respectively. Let $\widehat{f}$ be the Fourier transform of $f$ in both $x$ and $t$ variables

$$
\widehat{f}(\tau, \xi)=(2 \pi)^{-1} \iint_{\mathbb{R}^{2}} e^{-i t \tau-i x \xi} f(t, x) d t d x .
$$

Let $\langle\langle f, g\rangle\rangle$ and $f * g$ be the dual coupling and convolution of space and time variables respectively. Lastly for $-1<b<1$ we let $X_{b}$ and $Y_{b}$ denote the Hilbert spaces with norms

$$
\begin{gathered}
\|f\|_{X_{b}}=\left(\iint\left(1+\left|\tau+\xi^{2}\right|\right)^{2 b}|\widehat{f}(\tau, \xi)|^{2} d \tau d \xi\right)^{1 / 2} \\
\|g\|_{Y_{b}}=\left(\iint\left(1+\left|\tau-\xi^{3}\right|\right)^{2 b}(1+|\xi|)^{-1}|\widehat{g}(\tau, \xi)|^{2} d \tau d \xi\right)^{1 / 2} .
\end{gathered}
$$

As in [14] we observe that $\|f\|_{X_{b}}=\left\|\left(1+D_{t}\right)^{b} U(t) f\right\|_{L_{t}^{2}\left(L_{x}^{2}\right)}$ and $\|g\|_{Y_{b}}=$ $\left\|\left(1+D_{t}\right)^{b} V(t) g\right\|_{L_{t}^{2}\left(H_{x}^{-1 / 2}\right)}$, where $U(t)=e^{i t \partial_{x}^{2}}$ and $V(t)=e^{-t \partial_{x}^{3}}$.

Our theorem is as follows:

Theorem 1.1. For any $\left(u_{0}, v_{0}\right) \in L^{2}(\mathbb{R}) \times H^{-1 / 2}(\mathbb{R})$ and $b \in(1 / 2,7 / 12)$, there exist $T=T\left(\left\|u_{0}\right\|_{2},\left\|v_{0}\right\|_{H^{-1 / 2}}\right)>0$ and a unique solution $(u(t), v(t))$ of the initial value problem (1.2) satisfying

$$
\begin{aligned}
& u \in C\left([0, T) ; L^{2}(\mathbb{R})\right), v \in C\left([0, T) ; H^{-1 / 2}(\mathbb{R})\right), \\
& u \in X_{b}, v \in Y_{b}
\end{aligned}
$$

with

$$
u v,|u|^{2} u \in X_{b-1}, \partial_{x} v^{2}, \partial_{x}|u|^{2} \in Y_{b-1} .
$$

Moreover, given $T^{\prime} \in(0, T)$ the map $\left(u_{0}, v_{0}\right) \rightarrow(u(t), v(t))$ is Lipschitz continuous from $L^{2}(\mathbb{R}) \times H^{-1 / 2}(\mathbb{R})$ to $C\left(\left[0, T^{\prime}\right) ; L^{2}(\mathbb{R})\right) \times C\left(\left[0, T^{\prime}\right) ; H^{-1 / 2}(\mathbb{R})\right)$.

As a corollary of the above theorem, we can generalize the former result found in $[23]$.

Corollary 1.2. For any $s>0$ and $\left(u_{0}, v_{0}\right) \in H^{s}(\mathbb{R}) \times H^{s-1 / 2}(\mathbb{R})$, the same conclusion holds for $u \times v \in C\left([0, T) ; H^{s}(\mathbb{R})\right) \times C\left([0, T) ; H^{s-1 / 2}(\mathbb{R})\right)$.

Remark. The additional regularity (1.4) assures that the nonlinear terms in the integral equations have meaning.

Related to another kind of nonlinear Schrödinger equation, it should be commented that the weak solution for the so-called derivative nonlinear Schrödinger equation uniquely exists in $H^{1}(\mathbb{R})$ (see Hayashi [10], Hayashi-Ozawa [11] and Ozawa [21]). Those results are obtained by reducing the single equation into a system of 
nonlinear Schrödinger equations. Thanks to the stronger smoothing effect in the $\mathrm{KdV}$ part, our result can be handled with much weaker initial data.

Since the $L^{2}$ conservation law is established for the regular solutions ([23]), the solution obtained in the above also satisfies the $L^{2}$ conservation law for the Schrödinger part $u$. However since any a priori estimate for the KdV part is not known, it is open whether the solution in our theorem is globally well-posed or not.

\section{Preliminary estimates}

In what follows $\psi(t)$ denotes a cut off function in $C_{0}^{\infty}(\mathbb{R})$ such that it is 1 on the interval $[-1,1]$ and is 0 outside of $[-2,2]$. We let $\psi_{\delta}=\psi(t / \delta)$. First we state some elementary bounds involving the function spaces $X_{b}$ and $Y_{b}$ which are found in [14], [15].

Lemma 2.1 ([14], [15]). Let $b \in(1 / 2,1)$ and $\delta \in(0,1)$; then for $F \in X_{b}$ we have

$$
\begin{gathered}
\left\|\psi_{\delta} F\right\|_{X_{b}} \leq C \delta^{(1-2 b) / 2}\|F\|_{X_{b}} \quad \text { for } F \in X_{b}, \\
\left\|\psi_{\delta} F\right\|_{Y_{b}} \leq C \delta^{(1-2 b) / 2}\|F\|_{Y_{b}} \quad \text { for } F \in Y_{b} .
\end{gathered}
$$

For $b, b^{\prime} \in(0,1 / 2)$ with $b^{\prime}<b$ and $\delta \in(0,1)$, then for $F \in X_{-b^{\prime}}$ we have

$$
\begin{aligned}
& \left\|\psi_{\delta} F\right\|_{X_{-b}} \leq C \delta^{\left(b^{\prime}-b\right) / 4\left(1-b^{\prime}\right)}\|F\|_{X_{-b^{\prime}}} \quad \text { for } F \in X_{-b^{\prime}}, \\
& \left\|\psi_{\delta} F\right\|_{Y_{-b}} \leq C \delta^{\left(b^{\prime}-b\right) / 4\left(1-b^{\prime}\right)}\|F\|_{Y_{-b^{\prime}}} \quad \text { for } F \in Y_{-b^{\prime}} .
\end{aligned}
$$

Next we show the linear estimates needed for the linear parts of the Schrödinger equation and the KdV equation which are due to Kenig-Ponce-Vega [14] and [16]. Recall that $U(t)=e^{i t \partial_{x}^{2}}$ and $V(t)=e^{-t \partial_{x}^{3}}$ denote the linear Schrödinger and Airy unitary groups respectively.

Proposition 2.2 ([4], [14], [16]). Let $b \in(1 / 2,1)$ and $\delta \in(0,1)$; then we have

$$
\begin{gathered}
\left\|\psi_{\delta} U(t) u_{0}\right\|_{X_{b}} \leq C_{0} \delta^{(1-2 b) / 2}\left\|u_{0}\right\|_{2}, \\
\left\|\psi_{\delta} \int_{0}^{t} U\left(t-t^{\prime}\right) F\left(t^{\prime}\right) d t^{\prime}\right\|_{X_{b}} \leq C \delta^{(1-2 b) / 2}\|F\|_{X_{b-1}}, \\
\left\|\psi_{\delta} \int_{0}^{t} U\left(t-t^{\prime}\right) F\left(t^{\prime}\right) d t^{\prime}\right\|_{L^{\infty}\left((0, T) ; L^{2}\right)} \leq C \delta^{(1-2 b) / 2}\|F\|_{X_{b-1}} .
\end{gathered}
$$

Proposition 2.3 ([6], [14]). Let $b \in(1 / 2,1)$ and $\delta \in(0,1)$; then we have

$$
\begin{gathered}
\left\|\psi_{\delta} V(t) v_{0}\right\|_{Y_{b}} \leq C_{0} \delta^{(1-2 b) / 2}\left\|v_{0}\right\|_{H^{-1 / 2}}, \\
\left\|\psi_{\delta} \int_{0}^{t} V\left(t-t^{\prime}\right) F\left(t^{\prime}\right) d t^{\prime}\right\|_{Y_{b}} \leq C \delta^{(1-2 b) / 2}\|F\|_{Y_{b-1}}, \\
\left\|\psi_{\delta} \int_{0}^{t} V\left(t-t^{\prime}\right) F\left(t^{\prime}\right) d t^{\prime}\right\|_{L^{\infty}\left((0, T) ; H^{-1 / 2}\right)} \leq C \delta^{(1-2 b) / 2}\|F\|_{Y_{b-1}} .
\end{gathered}
$$

The following estimate due to Strichartz [22] is well-known and used often in the various areas of the study of nonlinear Schrödinger equations.

Proposition $2.4([22])$. Let $u_{0} \in L^{2}(\mathbb{R})$; then

$$
\left\|U(t) u_{0}\right\|_{L_{t}^{6}\left(L_{x}^{6}\right)} \leq C\left\|u_{0}\right\|_{2} .
$$


Finally we give some elementary estimates needed for the nonlinear estimates in Section 3.

Lemma 2.5. For $a, b>0$ and $\kappa=\min (a, b)$ with $a+b>1+\kappa$, there exists $C>0$ such that

$$
\int \frac{d x}{(1+|x-p|)^{a}(1+|x-q|)^{b}} \leq \frac{C}{(1+|p-q|)^{\kappa}} .
$$

For $a>1$ we have

$$
\int \frac{d x}{(1+|p x-q|)^{a}} \leq \frac{C}{|p|}
$$

For $a>1 / 3$ we have

$$
\int \frac{d x}{\left(1+\left|a_{0}+a_{1} x+a_{2} x^{2}+x^{3}\right|\right)^{a}} \leq C .
$$

Proof. Estimate (2.12) and (2.13) follow from simple calculus. We show the proof for (2.14).

$$
\begin{aligned}
& \int \frac{d x}{\left(1+\left|a_{0}+a_{1} x+a_{2} x^{2}+x^{3}\right|\right)^{a}} \\
& =\int_{B} \frac{d x}{\left.\left(1+\left|x-r_{1}\right|\left|x-r_{2}\right| \mid x-r_{3}\right) \mid\right)^{a}}+\int_{B^{c}} \frac{d x}{\left(1+\left|x-r_{1}\right|\left|x-r_{2}\right|\left|x-r_{3}\right|\right)^{a}},
\end{aligned}
$$

where $r_{1}, r_{2}$, and $r_{3}$ are the roots of the polynomial $a_{0}+a_{1} x+a_{2} x^{2}+x^{3}$ and $B$ is the union of three balls of radius 1 about the roots $r_{i}$.

Noting

$$
\left(1+\left|x-r_{1}\right|\left|x-r_{2}\right|\left|x-r_{3}\right|\right) \geq \frac{1}{7}\left(1+\left|x-r_{1}\right|\right)\left(1+\left|x-r_{2}\right|\right)\left(1+\left|x-r_{3}\right|\right)
$$

on $B^{c}$, we have

$$
\begin{aligned}
\int_{B^{c}} & \frac{d x}{\left(1+\left|x-r_{1}\right|\left|x-r_{2}\right|\left|x-r_{3}\right|\right)^{a}} \\
& \leq C \int_{B^{c}} \frac{d x}{\left(1+\left|x-r_{1}\right|\right)^{a}\left(1+\left|x-r_{2}\right|\right)^{a}\left(1+\left|x-r_{3}\right|\right)^{a}} .
\end{aligned}
$$

Using Hölder's inequality yields the result.

\section{NONLINEAR ESTIMATES}

Here we give four estimates for the nonlinear terms that are needed to complete the construction of local solutions with the use of the contraction mapping argument. First we treat the cubic nonlinear term in the Schrödinger part of the system.

Lemma 3.1. For $b, b^{\prime} \in(1 / 2,1)$ we have that

$$
\left\||u|^{2} u\right\|_{X_{b^{\prime}-1}} \leq C\|u\|_{X_{b}}^{3} .
$$


Proof. It is sufficient to show (3.1) for $u \in \mathcal{S}\left(\mathbb{R}^{2}\right)$. For $b, b^{\prime} \in(1 / 2,1)$,

$$
\begin{aligned}
\left\||u|^{2} u\right\|_{X_{b^{\prime}-1}} & =\left\|\left(1+\left|\tau+\xi^{2}\right|\right)^{b^{\prime}-1} \widehat{\left(|u|^{2} u\right)}\right\|_{L_{\tau}^{2}\left(L_{\xi}^{2}\right)} \\
& \leq \sup _{\xi, \tau}\left(1+\left|\tau+\xi^{2}\right|\right)^{b^{\prime}-1}\left\||u|^{2} u\right\|_{L_{t}^{2}\left(L_{x}^{2}\right)} \\
& \leq\|u\|_{L_{t}^{6}\left(L_{x}^{6}\right)}^{3} .
\end{aligned}
$$

Now let $f(\tau, \xi)=\left(1+\left|\tau+\xi^{2}\right|\right)^{b} \widehat{u}(\tau, \xi)$; then we have by the change of variables $\sigma=\tau+\xi^{2}$,

$$
\begin{aligned}
\|u\|_{L_{t}^{6}\left(L_{x}^{6}\right)} & =\left\|\iint e^{i x \xi} e^{i t \tau} \frac{f}{\left(1+\left|\tau+\xi^{2}\right|\right)^{b}} d \xi d \tau\right\|_{L_{t}^{6}\left(L_{x}^{6}\right)} \\
& =\left\|\int\left\{\frac{e^{i t \sigma}}{(1+|\sigma|)^{b}}\left(\int e^{i x \xi+i t \xi^{2}} f\left(\sigma+\xi^{2}, \xi\right) d \xi\right)\right\} d \sigma\right\|_{L_{t}^{6}\left(L_{x}^{6}\right)} .
\end{aligned}
$$

Set $f\left(\sigma-\xi^{2}, \xi\right)$ as $\widehat{g_{\sigma}}(\xi)$. Then

$$
\int e^{i x \xi+i t \xi^{2}} f\left(\sigma-\xi^{2}, \xi\right) d \xi=U(t) g_{\sigma}
$$

By applying the Strichartz estimate (2.11) to the above, we proceed

$$
\begin{aligned}
& \int\left\|\frac{e^{i t \sigma}}{(1+|\sigma|)^{b}} U(t) g_{\sigma}\right\|_{L_{t}^{6}\left(L_{x}^{6}\right)} d \sigma \leq \int \frac{1}{(1+|\sigma|)^{b}}\left\|U(t) g_{\sigma}\right\|_{L_{t}^{6}\left(L_{x}^{6}\right)} d \sigma \\
& \leq C\left\|\frac{1}{(1+|\sigma|)^{b}}\right\|_{L_{\sigma}^{2}}\left\|g_{\sigma}(x)\right\|_{L_{\sigma}^{2}\left(L_{x}^{2}\right)} \leq C\left\|g_{\sigma}(x)\right\|_{L_{\sigma}^{2}\left(L_{x}^{2}\right)} .
\end{aligned}
$$

Finally noting

$$
\left\|g_{\sigma}(x)\right\|_{L_{\sigma}^{2}\left(L_{x}^{2}\right)}=\|f\|_{L_{\tau}^{2}\left(L_{\xi}^{2}\right)}
$$

in (3.4), we obtain the conclusion from (3.2).

The following lemma is originally due to Bourgain ([6]) yet the techniques are established by Kenig-Ponce-Vega [15], which shows the remaining nonlinear estimates. The estimates for the interaction terms, (3.6) and (3.7), are new.

Lemma $3.2([15])$. For any $b_{1}, b_{1}^{\prime} \in(1 / 2,7 / 12), b_{2}, b_{2}^{\prime} \in(1 / 2,1)$ and $b_{3}, b_{3}^{\prime} \in$ $(1 / 2,3 / 4)$ there is a constant $C>0$ such that

$$
\begin{aligned}
& \left\|\partial_{x} v^{2}\right\|_{Y_{b_{1}^{\prime}-1}} \leq C\|v\|_{Y_{b_{1}}}^{2}, \\
& \left\|\partial_{x}|u|^{2}\right\|_{Y_{b_{2}^{\prime}-1}} \leq C\|u\|_{X_{b_{2}}}^{2}, \\
& \|u v\|_{X_{b_{3}^{\prime}-1}} \leq C\|u\|_{X_{b_{3}}}\|v\|_{Y_{b_{2}}} .
\end{aligned}
$$

Proof. The estimate (3.5) has been proved while establishing the local wellposedness of the KdV equation by Kenig-Ponce-Vega [15]. 
To prove inequality (3.6) we let $f(\tau, \xi)=\left(1+\left|\tau+\xi^{2}\right|\right)^{b_{1}} \widehat{u}(\tau, \xi)$ and $f^{*}(\tau, \xi)=$ $\left(1+\left|\tau-\xi^{2}\right|\right)^{b_{1}} \widehat{\bar{u}}(\tau, \xi)$ to obtain the following:

$$
\begin{aligned}
\| \partial_{x}|u|^{2} & \left\|_{Y_{b_{2}^{\prime}-1}}=\right\|\left(1+\left|\tau-\xi^{3}\right|\right)^{b_{2}^{\prime}-1}(1+|\xi|)^{-1 / 2} \widehat{\partial_{x}|u|^{2}} \|_{L_{\tau}^{2}\left(L_{\xi}^{2}\right)} \\
= & \left\|\frac{i \xi}{\left(1+\left|\tau-\xi^{3}\right|\right)^{1-b_{2}^{\prime}}(1+|\xi|)^{1 / 2}} \widehat{u} * \widehat{\bar{u}}\right\|_{L_{\tau}^{2}\left(L_{\xi}^{2}\right)} \\
\leq & \left\|\frac{|\xi|^{1 / 2}}{\left(1+\left|\tau-\xi^{3}\right|\right)^{1-b_{2}^{\prime}}}\left|\frac{f}{\left(1+\left|\tau+\xi^{2}\right|\right)^{b_{1}}} * \frac{f^{*}}{\left(1+\left|\tau-\xi^{2}\right|\right)^{b_{1}}}\right|\right\|_{L_{\tau}^{2}\left(L_{\xi}^{2}\right)} \\
\leq & \| \frac{|\xi|^{1 / 2}}{\left(1+\left|\tau-\xi^{3}\right|\right)^{1-b_{2}^{\prime}}} \\
& \times\left(\iint \frac{d \tau_{1} d \xi_{1}}{\left(1+\left|\tau_{1}+\xi_{1}^{2}\right|\right)^{2 b_{1}}\left(1+\left|\tau-\tau_{1}-\left(\xi-\xi_{1}\right)^{2}\right|\right)^{2 b_{1}}}\right)^{1 / 2} \\
& \times\left(|f|^{2} *\left|f^{*}\right|^{2}\right)^{1 / 2} \|_{L_{\tau}^{2}\left(L_{\xi}^{2}\right)} \\
\leq & \|h(\tau, \xi)\|_{L_{\tau}^{\infty}\left(L_{\xi}^{\infty}\right)}\|f\|_{L_{\tau}^{2}\left(L_{\xi}^{2}\right)}\left\|f^{*}\right\|_{L_{\tau}^{2}\left(L_{\xi}^{2}\right)} .
\end{aligned}
$$

Lemma 2.5, (2.12) and (2.13) yield

$$
\begin{aligned}
& |h(\tau, \xi)| \\
& \quad=\left|\frac{|\xi|^{1 / 2}}{\left(1+\left|\tau-\xi^{3}\right|\right)^{1-b_{2}^{\prime}}}\left(\iint \frac{d \tau_{1} d \xi_{1}}{\left(1+\left|\tau_{1}+\xi_{1}^{2}\right|\right)^{2 b_{1}}\left(1+\left|\tau-\tau_{1}-\left(\xi-\xi_{1}\right)^{2}\right|\right)^{2 b_{1}}}\right)^{1 / 2}\right| \\
& \quad \leq \frac{C|\xi|^{1 / 2}}{\left(1+\left|\tau-\xi^{3}\right|\right)^{1-b_{2}^{\prime}}}\left(\int \frac{d \xi_{1}}{\left(1+\left|\tau+\xi^{2}-2 \xi_{1} \xi\right|\right)^{2 b_{1}}}\right)^{1 / 2} \leq C,
\end{aligned}
$$

which shows (3.6).

Next we show (3.7). Let $u \in X_{b_{3}}$ and $v \in Y_{b_{2}}$. Again by the Plancharel identity,

$$
\begin{aligned}
\|u v\|_{X_{b_{3}^{\prime}-1}} & =\left\|\left(1+\left|\tau-\xi^{2}\right|\right)^{b_{3}^{\prime}-1} \widehat{u v}\right\|_{L_{\tau}^{2}\left(L_{\xi}^{2}\right)} \\
& =\sup _{\phi \in L_{\tau}^{2}\left(L_{\xi}^{2}\right),\|\phi\|_{2} \leq 1}\left|\left\langle\left\langle\frac{1}{\left(1+\left|\tau-\xi^{2}\right|\right)^{1-b_{3}^{\prime}}} \widehat{u} * \widehat{v}, \phi\right\rangle\right\rangle\right| .
\end{aligned}
$$

Letting $f(\tau, \xi)=\left(1+\left|\tau+\xi^{2}\right|\right)^{b_{3}} \widehat{u}(\tau, \xi)$ and $g(\tau, \xi)=\left(1+\left|\tau-\xi^{3}\right|\right)^{b_{2}}(1+|\xi|)^{-1 / 2} \widehat{v}(\tau, \xi)$, we compute (3.9) in the following way:

$$
\begin{aligned}
& \left\langle\left\langle\frac{1}{\left(1+\left|\tau+\xi^{2}\right|\right)^{1-b_{3}^{\prime}}} \widehat{u} * \widehat{v}, \bar{\phi}\right\rangle\right\rangle \\
& =\left\langle\left\langle\frac{1}{\left(1+\left|\tau+\xi^{2}\right|\right)^{1-b_{3}^{\prime}}}\left(\frac{f}{\left(1+\left|\tau+\xi^{2}\right|\right)^{b_{3}}} * \frac{(1+|\xi|)^{1 / 2} g}{\left(1+\left|\tau-\xi^{3}\right|\right)^{b_{2}}}\right), \bar{\phi}\right\rangle\right\rangle \\
& =\iiint \int \frac{\left(1+\left|\xi_{1}\right|\right)^{1 / 2} g\left(\tau_{1}, \xi_{1}\right) f\left(\tau-\tau_{1}, \xi-\xi_{1}\right) \bar{\phi}(\tau, \xi) d \tau_{1} d \xi_{1} d \tau d \xi}{\left(1+\left|\tau+\xi^{2}\right|\right)^{1-b_{3}^{\prime}}\left(1+\left|\tau_{1}-\xi_{1}^{3}\right|\right)^{b_{2}}\left(1+\left|\tau-\tau_{1}+\left(\xi-\xi_{1}\right)^{2}\right|\right)^{b_{3}}} \\
& =\iiint \int_{R_{1}}+\iiint \int_{R_{2}}+\iiint \int_{R_{3}} \equiv I_{1}+I_{2}+I_{3},
\end{aligned}
$$

where the integral region $\mathbb{R}^{4}$ is separated into three parts, $R_{1} \cup R_{2} \cup R_{3}$. Let $\chi_{R_{i}}$ be the characteristic function for each region $R_{i}(i=1,2,3)$. Then for the first part, 
we integrate over $\tau_{1}$ and $\xi_{1}$ and use Hölder's and Cauchy-Schwarz's inequality to obtain the following:

$$
\begin{aligned}
& \left|I_{1}\right| \leq\|\phi\|_{L_{\tau}^{2}\left(L_{\xi}^{2}\right)} \| \frac{1}{\left(1+\left|\tau+\xi^{2}\right|\right)^{1-b_{3}^{\prime}}} \\
& \times \iint \frac{\left(1+\left|\xi_{1}\right|\right)^{1 / 2} g\left(\tau_{1}, \xi_{1}\right) f\left(\tau-\tau_{1}, \xi-\xi_{1}\right) \chi_{R_{1}} d \xi_{1} d \tau_{1}}{\left(1+\left|\tau_{1}-\xi_{1}^{3}\right|\right)^{b_{2}}\left(1+\left|\tau-\tau_{1}+\left(\xi-\xi_{1}\right)^{2}\right|\right)^{b_{3}}} \|_{L_{\tau}^{2}\left(L_{\xi}^{2}\right)} \\
& \leq C\|\phi\|_{L_{\tau}^{2}\left(L_{\xi}^{2}\right)}\|g\|_{L_{\tau}^{2}\left(L_{\xi}^{2}\right)}\|f\|_{L_{\tau}^{2}\left(L_{\xi}^{2}\right)} \| \frac{1}{\left(1+\left|\tau+\xi^{2}\right|\right)^{1-b_{3}^{\prime}}} \\
& \times\left(\iint \frac{\left(1+\left|\xi_{1}\right|\right) \chi_{R_{1}} d \tau_{1} d \xi_{1}}{\left(1+\left|\tau_{1}-\xi_{1}^{3}\right|\right)^{2 b_{2}}\left(1+\left|\tau-\tau_{1}+\left(\xi-\xi_{1}\right)^{2}\right|\right)^{2 b_{3}}}\right)^{1 / 2} \|_{L_{\tau}^{\infty}\left(L_{\xi}^{\infty}\right)} \\
& \leq C\|u\|_{X_{b_{3}}}\|v\|_{Y_{b_{2}}} \| \frac{1}{\left(1+\left|\tau+\xi^{2}\right|\right)^{1-b_{3}^{\prime}}} \\
& \times\left(\iint \frac{\left(1+\left|\xi_{1}\right|\right) \chi_{R_{1}} d \tau_{1} d \xi_{1}}{\left(1+\left|\tau_{1}-\xi_{1}^{3}\right|\right)^{2 b_{2}}\left(1+\left|\tau-\tau_{1}+\left(\xi-\xi_{1}\right)^{2}\right|\right)^{2 b_{3}}}\right)^{1 / 2} \|_{L_{\tau}^{\infty}\left(L_{\xi}^{\infty}\right)} .
\end{aligned}
$$

Next for the second part, we integrate over $\tau$ and $\xi$ first and use the same steps as before to obtain the following:

$$
\begin{aligned}
\left|I_{2}\right| \leq & C\|\phi\|_{L_{\tau}^{2}\left(L_{\xi}^{2}\right)}\|g\|_{L_{\tau}^{2}\left(L_{\xi}^{2}\right)}\left\|\overline{f_{-}}\right\|_{L_{\tau}^{2}\left(L_{\xi}^{2}\right)} \\
\times & \times \frac{\left(1+\left|\xi_{1}\right|\right)^{1 / 2}}{\left(1+\left|\tau_{1}-\xi_{1}^{3}\right|\right)^{b_{2}}} \\
& \times\left(\iint \frac{\chi_{R_{2}} d \xi d \tau}{\left(1+\left|\tau+\xi^{2}\right|\right)^{2\left(1-b_{3}^{\prime}\right)}\left(1+\left|\tau-\tau_{1}+\left(\xi-\xi_{1}\right)^{2}\right|\right)^{2 b_{3}}}\right)^{1 / 2} \|_{L_{\tau_{1}\left(L_{\xi_{1}}^{\infty}\right)}} \\
\leq & C\|u\|_{X_{b_{3}}}\|v\|_{Y_{b_{2}}} \| \frac{\left(1+\left|\xi_{1}\right|\right)^{1 / 2}}{\left(1+\left|\tau_{1}-\xi_{1}^{3}\right|\right)^{b_{2}}} \\
& \times\left(\iint \frac{\chi_{R_{2}} d \xi d \tau}{\left(1+\left|\tau+\xi^{2}\right|\right)^{2\left(1-b_{3}^{\prime}\right)}\left(1+\left|\tau-\tau_{1}+\left(\xi-\xi_{1}\right)^{2}\right|\right)^{2 b_{3}}}\right)^{1 / 2} \|_{L_{\tau_{1}}^{\infty}\left(L_{\xi_{1}}^{\infty}\right)},
\end{aligned}
$$

where we put $f_{-}(\tau, \xi)=f(-\tau,-\xi)$. Note that $\left\|\overline{f_{-}}\right\|_{L_{\tau}^{2}\left(L_{\xi}^{2}\right)}=\|f\|_{L_{\tau}^{2}\left(L_{\xi}^{2}\right)}=\|u\|_{X_{b_{3}}}$.

For $I_{3}$, we use the change of variables $\tau_{1}-\tau=\tau_{2}$ and $\xi_{1}-\xi=\xi_{2}$ in (3.10) and obtain the bound:

$$
\begin{aligned}
\left|I_{3}\right| \leq C\|u\|_{X_{b_{3}}}\|v\|_{Y_{b_{2}}} \| \frac{1}{\left(1+\left|\tau_{2}-\xi_{2}^{2}\right|\right)^{b_{3}}} \\
\quad \times\left(\iint \frac{\left(1+\left|\xi_{1}\right|\right) \chi_{\tilde{R}_{3}} d \tau_{1} d \xi_{1}}{\left(1+\left|\tau_{1}-\tau_{2}+\left(\xi_{1}-\xi_{2}\right)^{2}\right|\right)^{2\left(1-b_{3}^{\prime}\right)}\left(1+\left|\tau_{1}-\xi_{1}^{3}\right|\right)^{2 b_{2}}}\right)^{1 / 2} \|_{L_{\tau_{2}\left(L_{\xi_{2}}^{\infty}\right)}},
\end{aligned}
$$

where $\tilde{R}_{3}$ is the region corresponding to $R_{3}$ in the variables $\left(\tau_{1}, \tau_{2}, \xi_{1}, \xi_{2}\right)$.

We then define the regions $R_{1}, R_{2}$, and $R_{3}$ such that $\mathbb{R}^{4}=R_{1} \cup R_{2} \cup R_{3}$. First we split $\mathbb{R}^{4}$ into three regions, $A, B$, and $C$ :

$$
\begin{aligned}
& A=\left\{\left(\tau, \tau_{1}, \xi, \xi_{1}\right) \in \mathbb{R}^{4}:\left|\xi_{1}\right| \leq 2\right\}, \\
& B=\left\{\left(\tau, \tau_{1}, \xi, \xi_{1}\right) \in \mathbb{R}^{4}:\left|\xi_{1}\right|>2 \text { and }\left|3 \xi_{1}^{2}-2 \xi_{1}+2 \xi\right| \geq\left|\xi_{1}\right|\right\}, \\
& C=\left\{\left(\tau, \tau_{1}, \xi, \xi_{1}\right) \in \mathbb{R}^{4}:\left|\xi_{1}\right|>2 \text { and }\left|\xi_{1}^{2}-\xi_{1}+2 \xi\right| \geq\left|\xi_{1}\right|\right\} .
\end{aligned}
$$


Since

$$
\begin{aligned}
& \left\{\left(\tau, \tau_{1}, \xi, \xi_{1}\right) \in \mathbb{R}^{4}:\left|\xi_{1}\right|>2\right. \text { and } \\
& \left.\qquad\left|3 \xi_{1}^{2}-2 \xi_{1}+2 \xi\right| \leq\left|\xi_{1}\right| \text { and }\left|\xi_{1}^{2}-\xi_{1}+2 \xi\right| \leq\left|\xi_{1}\right|\right\}
\end{aligned}
$$

is empty, we have $\mathbb{R}^{4}=A \cup B \cup C$.

Noting that for any points in $C$, we have that

$$
\left|\tau+\xi^{2}\right|+\left|\tau_{1}-\xi_{1}^{3}\right|+\left|\tau-\tau_{1}+\left(\xi-\xi_{1}\right)^{2}\right| \geq\left|\xi_{1}^{3}-\xi_{1}^{2}+2 \xi \xi_{1}\right| \geq\left|\xi_{1}\right|^{2},
$$

we further separate $C$ into three parts, i.e.,

$$
\begin{aligned}
& C_{1}=\left\{\left(\tau, \tau_{1}, \xi, \xi_{1}\right) \in C:\left|\tau_{1}-\xi_{1}^{3}\right|,\left|\tau-\tau_{1}+\left(\xi-\xi_{1}\right)^{2}\right| \leq\left|\tau+\xi^{2}\right|\right\}, \\
& C_{2}=\left\{\left(\tau, \tau_{1}, \xi, \xi_{1}\right) \in C:\left|\tau+\xi^{2}\right|,\left|\tau-\tau_{1}+\left(\xi-\xi_{1}\right)^{2}\right| \leq\left|\tau_{1}-\xi_{1}^{3}\right|\right\}, \\
& C_{3}=\left\{\left(\tau, \tau_{1}, \xi, \xi_{1}\right) \in C:\left|\tau+\xi^{2}\right|,\left|\tau_{1}-\xi_{1}^{3}\right| \leq\left|\tau-\tau_{1}+\left(\xi-\xi_{1}\right)^{2}\right|\right\}
\end{aligned}
$$

so that one of the following $\left|\tau+\xi^{2}\right|,\left|\tau_{1}-\xi_{1}^{3}\right|$, or $\left|\tau-\tau_{1}+\left(\xi-\xi_{1}\right)^{2}\right|$ is larger than $\left|\xi_{1}\right|^{2} / 3$.

We can now define the three sets that we separate $\mathbb{R}^{4}$ into:

$$
R_{1}=A \cup B \cup C_{1}, R_{2}=C_{2}, R_{3}=C_{3}
$$

and it is clear by construction that $\mathbb{R}^{4}=R_{1} \cup R_{2} \cup R_{3}$. Under the change of variables $\tau_{1}-\tau=\tau_{2}$ and $\xi_{1}-\xi=\xi_{2}$ the third region is transformed into

$$
\tilde{R}_{3}=\left\{\left(\tau_{1}, \tau_{2}, \xi_{1}, \xi_{2}\right) \in \mathbb{R}^{4}: 2<\left|\xi_{1}\right| \leq\left|\xi_{1}^{2}+\xi_{1}-2 \xi_{2}\right| \leq 3\left|\tau_{2}+\xi_{2}^{2}\right|\right\} .
$$

Thus, reviewing the estimates (3.11), (3.12) and (3.13), we will establish estimate (3.7) once the following lemma is shown.

Lemma 3.3. All the following expressions are bounded by a constant $C$ :

$$
\begin{aligned}
& \| \frac{1}{\left(1+\left|\tau+\xi^{2}\right|\right)^{1-b_{3}^{\prime}}} \\
& \quad \times\left(\iint \frac{\left(1+\left|\xi_{1}\right|\right) \chi_{R_{1}} d \tau_{1} d \xi_{1}}{\left(1+\left|\tau_{1}-\xi_{1}^{3}\right|\right)^{2 b_{2}}\left(1+\left|\tau-\tau_{1}+\left(\xi-\xi_{1}\right)^{2}\right|\right)^{2 b_{3}}}\right)^{1 / 2} \|_{L_{\tau}^{\infty}\left(L_{\xi}^{\infty}\right)} \leq C, \\
& \| \frac{\left(1+\left|\xi_{1}\right|\right)^{1 / 2}}{\left(1+\left|\tau_{1}-\xi_{1}^{3}\right|\right)^{b_{2}}} \\
& \quad \times\left(\iint \frac{\chi_{R_{2}} d \tau d \xi}{\left(1+\left|\tau+\xi^{2}\right|\right)^{2\left(1-b_{3}^{\prime}\right)}\left(1+\left|\tau-\tau_{1}+\left(\xi-\xi_{1}\right)^{2}\right|\right)^{2 b_{3}}}\right)^{1 / 2} \|_{L_{\tau_{1}}^{\infty}\left(L_{\xi_{1}}^{\infty}\right)} \leq C, \\
& \left\|\frac{1}{\left(1+\left|\tau_{2}-\xi_{2}^{2}\right|\right)^{b_{3}}} \quad \times\left(\iint \frac{\left(1+\left|\xi_{1}\right|\right) \chi_{\tilde{R}_{3}} d \tau_{1} d \xi_{1}}{\left(1+\left|\tau_{1}-\xi_{1}^{3}\right|\right)^{2 b_{2}}\left(1+\left|\tau_{1}-\tau_{2}+\left(\xi_{1}-\xi_{2}\right)^{2}\right|\right)^{2\left(1-b_{3}^{\prime}\right)}}\right)^{1 / 2}\right\|_{L_{\tau_{2}}^{\infty}\left(L_{\xi_{2}}^{\infty}\right)} \leq C .
\end{aligned}
$$


Proof of Lemma 3.3. According to Lemma 2.5, (2.12), it suffices to get bounds for

$$
\frac{1}{\left(1+\left|\tau+\xi^{2}\right|\right)^{1-b_{3}^{\prime}}}\left(\int \frac{\left(1+\left|\xi_{1}\right|\right)}{\left(1+\left|\tau+\xi^{2}-\xi_{1}^{3}+\xi_{1}^{2}-2 \xi \xi_{1}\right|\right)^{2 \kappa_{1}}} d \xi_{1}\right)^{1 / 2} \text { on } R_{1}
$$

$$
\frac{\left(1+\left|\xi_{1}\right|\right)^{1 / 2}}{\left(1+\left|\tau_{1}-\xi_{1}^{3}\right|\right)^{b_{2}}}\left(\int \frac{1}{\left(1+\left|\tau_{1}-\xi_{1}^{2}+2 \xi_{1} \xi\right|\right)^{2 \kappa_{2}}} d \xi\right)^{1 / 2} \text { on } R_{2}
$$

$$
\frac{1}{\left(1+\left|\tau_{2}-\xi_{2}^{2}\right|\right)^{b_{3}}}\left(\int \frac{\left(1+\left|\xi_{1}\right|\right)}{\left.\left(1+\mid \tau_{2}-\xi_{2}^{2}-\xi_{1}^{3}-\xi_{1}^{2}+2 \xi_{2} \xi_{1}\right)^{2} \mid\right)^{2 \kappa_{3}}} d \xi_{1}\right)^{1 / 2} \text { on } \tilde{R}_{3}
$$

where $\kappa_{1}=\min \left(b_{2}, b_{3}\right), \kappa_{2}=\min \left(1-b_{3}^{\prime}, b_{3}\right)$ and $\kappa_{3}=\min \left(b_{2}, 1-b_{3}^{\prime}\right)$.

We start with (3.15) in region $R_{1}=A \cup B \cup C_{1}$. In region $A$, we have $\left|\xi_{1}\right|<2$ and obviously

$$
\frac{1}{\left(1+\left|\tau+\xi^{2}\right|\right)^{1-b_{3}^{\prime}}}\left(\int \frac{\left(1+\left|\xi_{1}\right|\right) d \xi_{1}}{\left(1+\left|\tau+\xi^{2}-\xi_{1}^{3}+\xi_{1}^{2}-2 \xi \xi_{1}\right|\right)^{2 \kappa_{1}}}\right)^{1 / 2} \leq C .
$$

Next we estimate (3.15) in region $B$. By the change of variables $\eta=\tau-\xi_{1}^{3}+\xi_{1}^{2}-$ $2 \xi \xi_{1}+\xi^{2}$ and the condition $\left|\xi_{1}\right| \leq\left|3 \xi_{1}^{2}-2 \xi+2 \xi\right|$ on $B$, we obtain

$$
\begin{gathered}
\frac{1}{\left(1+\left|\tau+\xi^{2}\right|\right)^{1-b_{3}^{\prime}}}\left(\int \frac{\left|\xi_{1}\right| d \xi_{1}}{\left(1+\left|\tau+\xi^{2}-\xi_{1}^{3}+\xi_{1}^{2}-2 \xi \xi_{1}\right|\right)^{2 \kappa_{1}}}\right)^{1 / 2} \\
\leq C\left(\int \frac{d \eta}{(1+|\eta|)^{2 \kappa_{1}}}\right)^{1 / 2} \leq C .
\end{gathered}
$$

Note that $2 \kappa_{1}>1$. Similarly on region $C_{1}$, we have that $\left|\xi_{1}\right| \leq C\left(1+\left|\tau+\xi^{2}\right|\right)^{2\left(1-b_{3}^{\prime}\right)}$ for $b_{3}^{\prime} \leq 3 / 4$ and it follows from Lemma $2.5,(2.14)$ that

$$
\begin{aligned}
\frac{1}{\left(1+\left|\tau+\xi^{2}\right|\right)^{1-b_{3}^{\prime}}} & \left(\int \frac{\left(1+\left|\xi_{1}\right|\right) d \xi_{1}}{\left(1+\left|\tau+\xi^{2}-\xi_{1}^{3}+\xi_{1}^{2}-2 \xi \xi_{1}\right|\right)^{2 \kappa_{1}}}\right)^{1 / 2} \\
\leq & \left(\int \frac{\left|\xi_{1}\right| d \xi_{1}}{\left(1+\left|\tau+\xi^{2}\right|\right)^{2\left(1-b_{3}^{\prime}\right)}\left(1+\left|\tau+\xi^{2}-\xi_{1}^{3}+\xi_{1}^{2}-2 \xi \xi_{1}\right|\right)^{2 \kappa_{1}}}\right)^{1 / 2} \\
\leq & C\left(\int \frac{d \xi_{1}}{\left(1+\left|\tau+\xi^{2}-\xi_{1}^{3}+\xi_{1}^{2}-2 \xi \xi_{1}\right|\right)^{2 \kappa_{1}}}\right)^{1 / 2} \leq C .
\end{aligned}
$$

Thus gathering (3.18)-(3.20), we have shown (3.15). Next we show (3.16). Since $\frac{1}{2}-\kappa_{2}-b_{2}<0$, the change of variables $\eta=\tau_{1}+\xi_{1}^{2}-2 \xi \xi_{1}$, Lemma 2.5, (2.13) coupled with the restriction on the region with (3.14) yield the following:

$$
\begin{aligned}
\frac{\left|\xi_{1}\right|^{1 / 2}}{\left(1+\left|\tau_{1}-\xi_{1}^{3}\right|\right)^{b_{2}}} & \left(\int \frac{d \xi}{\left(1+\left|\tau_{1}+\xi_{1}^{2}-2 \xi \xi_{1}\right|\right)^{2 \kappa_{2}}}\right)^{1 / 2} \\
& \leq \frac{C}{\left(1+\left|\tau_{1}-\xi_{1}^{3}\right|\right)^{b_{2}}}\left(\int_{|\eta| \leq 4\left|\tau_{1}-\xi_{1}^{3}\right|} \frac{d \eta}{(1+|\eta|)^{2 \kappa_{2}}}\right)^{1 / 2} \\
& \leq C \frac{\left(1+\left|\tau_{1}-\xi_{1}^{3}\right|\right)^{1 / 2-\kappa_{2}}}{\left(1+\left|\tau_{1}-\xi_{1}^{3}\right|\right)^{b_{2}}} \leq C .
\end{aligned}
$$


Lastly on the region $\tilde{R}_{3}$ we note that $\left|\xi_{1}\right| /\left(1+\left|\tau_{2}-\xi_{2}^{2}\right|\right)^{2 b_{3}}<C$ and from Lemma $2.5,(2.14)$, we have that

$$
\begin{aligned}
\frac{1}{\left(1+\left|\tau_{2}-\xi_{2}^{2}\right|\right)^{b_{3}}}( & \left.\int \frac{\left(1+\left|\xi_{1}\right|\right) d \xi_{1}}{\left(1+\left|\tau_{2}-\xi_{2}^{2}+\xi_{1}^{3}-\xi_{1}^{2}-2 \xi_{2} \xi_{1}^{2}\right|\right)^{2 \kappa_{3}}}\right)^{1 / 2} \\
& \leq C\left(\int \frac{d \xi_{1}}{\left(1+\left|\tau_{2}-\xi_{2}^{2}-2 \xi_{1} \xi_{2}-\xi_{1}^{2}+\xi_{1}^{3}\right|\right)^{2 \kappa_{3}}}\right)^{1 / 2} \leq C .
\end{aligned}
$$

Here we have used that $\kappa_{3}>\frac{1}{6}$ and $b_{3} \geq \frac{1}{4}$. Now (3.15)-(3.17) are shown to be bounded and proof of Lemma 3.3 and, hence, the proof of Lemma 3.2 are completed.

The following lemma is an immediate consequence of Lemma 3.1 and Lemma 3.2 .

Lemma 3.4. Let $u, \tilde{u} \in X_{b_{1}}$ and $v, \tilde{v} \in Y_{b_{2}}$ with $b_{1}, b_{1}^{\prime} \in(1 / 2,3 / 4)$, and $b_{2}, b_{2}^{\prime} \in$ $(1 / 2,7 / 12)$. Then there is a constant $C>0$ such that

$$
\begin{aligned}
& \left\||u|^{2} u-|\tilde{u}|^{2} \tilde{u}\right\|_{X_{b_{1}^{\prime}-1}} \leq C\left(\|u\|_{X_{b_{1}}}^{2}+\|\tilde{u}\|_{X_{b_{1}}}^{2}\right)\|u-\tilde{u}\|_{X_{b_{1}}}, \\
& \|u v-\tilde{u} \tilde{v}\|_{X_{b_{1}^{\prime}-1}} \leq C\left(\|u-\tilde{u}\|_{X_{b_{1}}}\|v\|_{Y_{b_{2}}}+\|\tilde{u}\|_{X_{b_{1}}}\|v-\tilde{v}\|_{Y_{b_{2}}}\right), \\
& \left\|\partial_{x}|u|^{2}-\partial_{x}|\tilde{u}|^{2}\right\|_{Y_{b_{2}^{\prime}-1}} \leq C\left(\|u\|_{X_{b_{1}}}+\|\tilde{u}\|_{X_{b_{1}}}\right)\|u-\tilde{u}\|_{X_{b_{1}}}, \\
& \left\|\partial_{x} v^{2}-\partial_{x} \tilde{v}^{2}\right\|_{Y_{b_{2}^{\prime}-1}} \leq C\left(\|v\|_{Y_{b_{2}}}+\|\tilde{v}\|_{Y_{b_{2}}}\right)\|v-\tilde{v}\|_{Y_{b_{2}}} .
\end{aligned}
$$

\section{Proof of Theorem 1.1}

Now that the necessary estimates have been deduced the rest of the proof follows the arguments appearing in [14] (see also [16]). We give the outline of the arguments for completeness. Note that in our case, we cannot employ the scaling argument because of the presence of the pure power term in the Schrödinger part. Recall that $\psi$ is the smooth cut-off function as defined in the section 2 , where we denote $\psi_{\delta}=\psi(t / \delta)$ so that $\psi_{1}(t)=\psi(t)$.

Proof of Theorem 1.1. We consider the following function space where we seek our solution. For $\left(u_{0}, v_{0}\right) \in L^{2}(\mathbb{R}) \times H^{-1 / 2}(\mathbb{R})$ and $b \in(1 / 2,7 / 12)$, let

$$
\mathcal{X}_{M N}=\left\{(u, v): u \in X_{b}, v \in Y_{b} \text { such that }\|u\|_{X_{b}} \leq M,\|v\|_{Y_{b}} \leq N\right\},
$$

where $M=2 C_{0}\left\|u_{0}\right\|_{2}$ and $N=2 C_{0}\left\|v_{0}\right\|_{H^{-1 / 2}}$. Then $\mathcal{X}_{M N}$ is a complete metric space with norm

$$
\|(u, v)\|{\mathcal{\mathcal { X } _ { M N }}}=\|u\|_{X_{b}}+\|v\|_{Y_{b}} .
$$

Without loss of generality, we may assume that $1<M$ and $1<N$.

For $(u, v) \in \mathcal{X}_{M N}$, we define the maps,

$$
\begin{aligned}
& \Phi[u, v]=\psi_{1}(t) U(t) u_{0}-i \psi_{1}(t) \int_{0}^{t} U\left(t-t^{\prime}\right) \psi_{\delta}\left(t^{\prime}\right)\left\{\alpha u v\left(t^{\prime}\right)+\gamma|u|^{2} u\left(t^{\prime}\right)\right\} d t^{\prime} \\
& \Psi[u, v]=\psi_{1}(t) W(t) v_{0}+\psi_{1}(t) \int_{0}^{t} W\left(t-t^{\prime}\right) \psi_{\delta}\left(t^{\prime}\right)\left\{\beta \partial_{x}|u|^{2}\left(t^{\prime}\right)-\partial_{x} v^{2}\left(t^{\prime}\right)\right\} d t^{\prime}
\end{aligned}
$$


Then according to Lemma 2.1, Proposition 2.2, Proposition 2.3, Lemma 3.1 and Lemma 3.2, we have for $b<b^{\prime}<7 / 12$ and $\mu=\left(b^{\prime}-b\right) / 4 b^{\prime}$,

$$
\begin{aligned}
&\|\Phi[u, v]\|_{X_{b}} \leq C_{0}\left\|u_{0}\right\|_{2}+C\left\|\psi_{\delta}\left\{\alpha u v+\gamma|u|^{2} u\right\}\right\|_{X_{b-1}} \\
& \leq C_{0}\left\|u_{0}\right\|_{2}+C \delta^{\mu}\left(\|u v\|_{X_{b^{\prime}-1}}+\left\|\left.u\right|^{2} u\right\|_{X_{b^{\prime}-1}}\right) \\
& \leq C_{0}\left\|u_{0}\right\|_{2}+C \delta^{\mu}\left(\|u\|_{X_{b}}\|v\|_{Y_{b}}+\|u\|_{X_{b}}^{3}\right) \\
&\|\Psi[u, v]\|_{Y_{b}} \leq C_{0}\left\|v_{0}\right\|_{H^{-\frac{1}{2}}}+C\left\|\psi_{\delta}\left\{\beta \partial_{x}|u|^{2}-\partial_{x} v^{2}\right\}\right\|_{Y_{b-1}} \\
& \leq C_{0}\left\|v_{0}\right\|_{H^{-\frac{1}{2}}}+C \delta^{\mu}\left(\left\|\partial_{x}|u|^{2}\right\|_{Y_{b^{\prime}-1}}+\left\|\partial_{x} v^{2}\right\|_{Y_{b^{\prime}-1}}\right) \\
& \leq C_{0}\left\|v_{0}\right\|_{H^{-\frac{1}{2}}}+C \delta^{\mu}\left(\|u\|_{X_{b}}^{2}+\|v\|_{Y_{b}}^{2}\right) .
\end{aligned}
$$

It follows from (4.1) and (4.2) that

$$
\begin{aligned}
& \|\Phi[u, v]\|_{X_{b}} \leq \frac{M}{2}+C_{1} \delta^{\mu}\left(M^{3}+M N\right), \\
& \|\Psi[u, v]\|_{Y_{b}} \leq \frac{N}{2}+C_{2} \delta^{\mu}\left(M^{2}+N^{2}\right) .
\end{aligned}
$$

If we set

$$
\delta^{\mu} \leq \frac{1}{2 \max \left(C_{1}, C_{2}\right)\left(M^{2}+N\right)}
$$

then we have that $\|\Phi[u, v]\|_{X_{b}} \leq M$ and $\|\Psi[u, v]\|_{Y_{b}} \leq N$; hence $(\Phi[u, v], \Psi[u, v]) \in$ $\mathcal{X}_{M N}$.

Similarly by Lemma 3.4 we have that

$$
\begin{gathered}
\|\Phi[u, v]-\Phi[\tilde{u}, \tilde{v}]\|_{X_{b}} \leq \frac{1}{4}\left(\|u-\tilde{u}\|_{X_{b}}+\|v-\tilde{v}\|_{Y_{b}}\right), \\
\|\Psi[u, v]-\Psi[\tilde{u}, \tilde{v}]\|_{Y_{b}} \leq \frac{1}{4}\left(\|u-\tilde{u}\|_{X_{b}}+\|v-\tilde{v}\|_{Y_{b}}\right)
\end{gathered}
$$

if $\delta^{\mu} \leq\left(2 C_{1}\left(2 M^{2}+N\right)\right)^{-1}$.

Therefore the map $\Phi \times \Psi:(u, v) \rightarrow(\Phi[u, v], \Psi[u, v])$ is a contraction mapping from $\mathcal{X}_{M N}$ into itself and we obtain a unique fixed point which solves the equation for $T<\delta$. The additional regularity

$$
u \in C\left([0, T) ; L^{2}\right), \quad v \in C\left([0, T) ; H^{-1 / 2}\right)
$$

both follow from Proposition 2.2, (2.7), Proposition 2.3, (2.10), and the $L^{2}, H^{-1 / 2}$ boundedness of the unitary operators $U(t)$ and $V(t)$ and we complete the proof.

\section{REFERENCES}

1. Bekiranov, D., Ogawa, T., Ponce, G., On the well-posedness of Benney's interaction equation of short and long waves, Adv. Differential Equations 1 (1996), no. 6, 919-937. CMP 97:01

2. Benilov, E.S., Burtsev, S.P., To the integrability of the equations describing the Langmuirwave -ion-wave interaction, Phys. Let. 98A (1983) 256-258. MR 85f:76120

3. Benney, D. J., A general theory for interactions between short and long waves, Stud. Appl. Math. 56, (1977), 81-94. MR 57:3657

4. Bourgain, J., Fourier transform restriction phenomena for certain lattice subsets and applications to nonlinear evolution equations. I Schrödinger equations, Geometric and Funct. Anal. 3 (1993), 107-156. MR 95d:35160a

5. Bourgain, J.,Exponential sums and nonlinear Schrödinger equations, ibid. 3 (1993), 157-178. MR 95d:35159 
6. Bourgain, J., Fourier transform restriction phenomena for certain lattice subsets and applications to nonlinear evolution equations. II The KdV equation, ibid. 3 (1993), 209-262. MR 95d:35160b

7. Cazenave, T., Weissler, F. B., The Cauchy problem for the critical nonlinear Schrödinger equation in $H^{s}$, Nonlinear Anal. T.M.A., 100 (1990), 807-836. MR 91j:35252

8. Funakoshi, M. Oikawa, M., The resonant interaction between a long internal gravity wave and a surface gravity wave packet. J. Phys. Soc. Japan 52 (1983), 1982-1995. MR 84k:76030

9. Ginibre, J., Le problème de Cauchy pour des edp semi-linéaires périodiques en variables d'espace [d'apès Bourgain], Séminaire BOURBAKI, 47 (1994-5) 796, 1-23.

10. Hayashi, N., The initial value problem for the derivative nonlinear Schrödinger equation, Nonlinear Anal. TMA. 20 (1993), 823-833, MR 94c:35007

11. Hayashi, N., Ozawa, T., Modified wave operators for the derivative nonlinear Schrödinger equation, Math. Annalen 298(1994), 557-576, MR 95f:35240

12. Kato, T. On nonlinear Schrödinger equations, Ann. Inst. Henri Poincaré, Physique théorique, 46 (1987), 113-129. MR 88f:35133

13. Kawahara, T., Sugimoto, N., Kakutani, T., Nonlinear interaction between short and long capillary-gravity waves, J. Phys. Soc. Japan, 39 (1975), 1379-1386

14. Kenig, C. E., Ponce, G., Vega, L., The Cauchy problem for the Korteweg-de Vries equation in Sobolev spaces of negative indices, Duke Math. J. 71 (1993) 1-21. MR 94g:35196

15. Kenig, C. E., Ponce, G., Vega, L., A bilinear estimate with applications to the KdV equation. J. Amer. Math. Soc. 9 (1996) 573-603. MR 96k:35159

16. Kenig, C. E., Ponce, G., Vega, L., Quadratic Forms for the 1-D semilinear Schrödinger equation, Trans. Amer. Math. Soc. 348 (1996), no. 8, 3323-3353. MR 96j:35233

17. Klainerman, S., Machedon, M., On the Maxwell-Klein-Gordon equation with finite energy, to appear in Duke Math. J.

18. Laurençot, $\mathrm{Ph} .$, On a nonlinear Schrödinger equation arising in the theory of water waves, Nonlinear Anal. TMA 24 (1995), 509-527. MR 95k:35194

19. Ma, Yan-Chow, The complete solution of the long-wave-short -wave resonance equations, Stud. Appl. Math., 59 (1978), 201-221. MR 58:25366

20. Nishikawa, K., Hojo, H., Mima, K., Ikezi, H. Coupled nonlinear electron-plasma and ion acoustic waves, Phys. Rev. Lett. 33 (1974), 148-151.

21. Ozawa, T., On the nonlinear Schrödinger equations of derivative type, Indiana Univ. Math. J. 45 (1996), no. 1, 137-163. CMP 96:17

22. Strichartz, R. S., Restriction of Fourier transform to quadratic surfaces and decay of solutions of wave equation, Duke Math. J., 44, (1977), 705-714. MR 58:23577

23. Tsutsumi, M., Well-posedness of the Cauchy problem for a coupled Schrödinger-KdV equation, Math. Sci. Appl. 2 (1993) 513-528. MR 96k:35163

24. Tsutsumi, M., Hatano, S., Well-posedness of the Cauchy problem for Benney's first equations of long wave short wave interactions, Funkcialaj Ekvacioj, 37 (1994), 289-316 MR 95k:35196

25. Tsutsumi, Y., $L^{2}$ - solutions for nonlinear Schrödinger equations and nonlinear group, ibid., 30 (1987), 115-125. MR 89c:35143

26. Yajima, N., Oikawa, M., Formation and interaction of sonic Langmuir soliton, Prog. Theor. Phys. 56 (1974), 1719-1739. MR 55:11882

27. Yajima, N., Satsuma, J., Soliton solutions in a diatomic lattice system, ibid., 62 (1979), 370-378.

Department of Mathematics, Florida International University, Miami, Florida 33199

E-mail address: bekiranov@fiu.edu

Graduate School of Polymathematics, Nagoya University, Nagoya, 464-01 Japan

E-mail address: ogawa@math.nagoya-u.ac.jp

Department of Mathematics, University of California Santa Barbara, Santa BarBARA, CALIFORNIA 93106

E-mail address: ponce@math.ucsb.edu 